Enfermagem Brasil 2018;17(6):561-7

https://doi.org/10.33233/eb.v17i6.708

\title{
ARTIGO ORIGINAL \\ Dificuldades encontradas por auxiliares e técnicos de enfermagem para realização de cálculos de medicamentos
}

Marcio Antonio de Assis, D.Sc. ${ }^{*}$, Milaine Garcia de Assis, M.Sc. ${ }^{*}$, Jaqueline Terra da Silva***, Evilin Cristine Rodrigues ${ }^{\star * *}$, Larissa Conti Di Cianni Rodrigues ${ }^{\star * *}$, Thais Fátima de Matos ${ }^{\star \star *}$

*Enfermeiro, Docente do curso de graduação em Enfermagem da Universidade de Mogi das Cruzes (UMC), Mogi das Cruzes/SP, ${ }^{*}$ Enfermeira da Prefeitura Municipal de Guararema/SP, ***Graduanda do Curso de Enfermagem da Universidade de Mogi das Cruzes (UMC), Mogi das Cruzes/SP

Recebido em 17 de janeiro de 2017; aceito em 24 de julho de 2018.

Endereço de correspondência: Marcio Antonio de Assis, Av. Missionária, 540 Jardim Cacique 08616-220 Suzano SP, E-mail: marcioassis80@gmail.com; Milaine Garcia de Assis: milaine.assis@guarerama.sp.gov.br; Jaqueline Terra da Silva: jaquelineterra@gmail.com; Evilin Cristine Rodrigues: evilinrodrigues93@gmail.com; Larissa Conti Di Cianni Rodrigues: larirodrigues13@hotmail.com

\section{Resumo}

Objetivo: Identificar fatores que dificultam o entendimento do cálculo de medicamentos por auxiliares e técnicos de enfermagem e levantar as possibilidades de melhorias quanto a esse entendimento. Métodos: Trata-se de um estudo do tipo survey descritivo e exploratório, com abordagem quantitativa, realizado com 50 auxiliares e técnicos de enfermagem, atuantes em área hospitalar, com experiência no preparo e administração de medicamentos, por meio da aplicação de um questionário semiestruturado. Resultados: $O$ fator que mais dificulta a realização dos cálculos é a falta de conhecimento dos participantes em relação à matemática, bem como sobre a compreensão do que se necessita. Conclusão: Existe a necessidade de melhorar a clareza referente ao processo de medicação, bem como reforçar os programas de educação continuada no sentido de favorecer uma maior segurança e assertividade nesse processo na assistência e enfermagem.

Palavras-chave: erros de medicação, assistência de enfermagem, gestão de riscos.

\section{Abstract \\ Difficulties encountered by nursing auxiliaries and technicians to perform drug calculations}

Objective: To identify factors that makes it difficult for the nursing team to understand the calculation of medication and to raise the possibility of improvements regarding this understanding. Methods: This was a descriptive and exploratory study, with a quantitative approach, carried out with 50 nursing assistants and technicians working in a hospital area region, with experience in the preparation and administration of medications, through the application of a semi structured questionnaire. Results: The factor that makes calculations more difficult is the lack of knowledge of the participants in relation to mathematics, as well as the understanding of what is needed. Conclusion: There is a need to improve the clarity regarding the medication process, as well as to reinforce the programs of continuing education in favor of a greater safety and assertiveness in this process, together with care and nursing.

Key-words: medication errors, nursing care, risk management.

\section{Resumen}

Las dificultades encontradas por los auxiliares de enfermería y técnicos para llevar a cabo los cálculos de drogas

Objetivo: Identificar los factores que dificultan la comprensión del cálculo de medicación por el personal de enfermería y aumentar las posibilidades de mejorias en ese entendimiento. Métodos: Se realizó un estudio de tipo estudio exploratorio descriptivo, con enfoque cuantitativo, realizado con 50 auxiliares y técnicos de enfermería, que trabajan en el área hospitalaria, con 
experiencia en la preparación y administración de drogas a través de la aplicación de un cuestionario semi-estructurado. Resultados: El principal factor que dificulta la realización de los cálculos es la falta de conocimiento matemático de los participantes, así como la comprensión de lo que se necesita. Conclusión: Existe una necesidad de mejorar la claridad con respecto al proceso de medicación y fortalecer los programas de educación continuada con el fin de fomentar una mayor seguridad y asertividad en este proceso con la atención de enfermería.

Palabras-clave: errores de medicación, atención de enfermería, gestión de riesgo.

Introdução

Medicamentos são substâncias químicas que auxiliam na restauração e manutenção da saúde, atuando também na prevenção e na melhoria da qualidade de vida da população. A utilização indevida dos medicamentos, entretanto, constitui-se em uma das principais causas de complicações à saúde, especialmente no ambiente hospitalar [1].

A prática da administração de medicamentos é uma das atividades mais importantes realizada pela equipe de enfermagem e, por ser frequente, possui grande importância no contexto de segurança na assistência hospitalar. Ela inicia-se na padronização e compra dos medicamentos, seguido pela prescrição, transcrição, distribuição, preparo e administração [2].

Em uma unidade de saúde, esse processo contextualiza-se como um sistema multidisciplinar. Contudo, compete a enfermagem duas etapas importantes desse processo: 0 preparo e administração de medicamentos. Assim, é imprescindível conhecer a ação e os efeitos dos medicamentos, como também acompanhar e monitorar a resposta dos clientes com intuito de evitar eventos adversos [3]

Os eventos adversos podem ser designados como os efeitos indesejados e imprevistos, provenientes do processo de medicação, representando os riscos no uso de medicamento. Dentre esses riscos, existem os que são próprios da prática de medicação, no qual se incluem as reações adversas e aqueles que não são próprios, classificados como erros de medicação [4].

De acordo com a National Coordinating Council for Medication Error Reporting and Prevention [5], uma organização norte - americana independente, composta por 27 organizações internacionais e nacionais, o termo "erro de medicação" tem o seguinte significado: toda e qualquer situação que pode ser prevenida, mas em que o paciente pode ser prejudicado devido à falta de concentração do profissional que o manuseava.

$\mathrm{O}$ erro de medicação, independente da etapa em que ocorre, ocasionam danos à integridade e saúde do paciente, o que aumenta o seu tempo de internação, comprometendo a equipe multiprofissional e a instituição [2].

Os erros negligenciados e não detectados em etapas anteriores são atribuídos à enfermagem, uma vez que a medicação é uma parte integrante e fundamental da assistência, sendo responsabilidade do enfermeiro. Essa responsabilidade é, mais uma vez, destacada nos artigos do Código de Ética dos Profissionais de Enfermagem [6].

Uma ferramenta que auxilia e norteia a equipe de enfermagem na administração de medicamentos é o método dos cinco certos, criado na década de 60 como prática de verificação, classificados em: paciente certo, medicamento certo, dose certa, horário certo e via certa. Esses certos das medicações foram designados para garantir a segurança do paciente e prevenir danos [7].

Entretanto, o uso desses princípios não assegura o êxito da prática de medicação, sendo plausível a ocorrência de erros em qualquer fase desse processo. Um fator que favorece o erro de medicação é a dificuldade encontrada pela equipe de enfermagem em calcular precisamente a dose do medicamento a ser administrado [9].

Desse modo, pode-se identificar um empecilho ao seguimento das etapas que asseguram o modo correto de garantir a segurança no processo de aplicação medicamentosa. O profissional que não compreende ou possui dificuldade em calcular dosagens ou o fluxo de infusão, não possui condições de seguir o certo relacionado à dose.

Para que sejam implantadas ações multidisciplinares que tragam melhorias para as instituições de saúde é necessário conhecer e analisar os fatores que causam os erros de medicação e interferem na qualidade assistencial, minimizando, portanto, os erros dentro deste processo e padronizando assim, regras para toda a equipe envolvida [2]. Ao levantar as situações que comprometem o entendimento e aprendizagem do profissional de enfermagem sobre cálculos de medicações, será possível identificar estratégias para reverter ou minimizar essas condições. 
Diante disso, o objetivo do presente estudo foi identificar fatores que dificultam o entendimento do cálculo de medicamentos pela equipe de enfermagem e levantar as possibilidades de melhorias quanto a esse entendimento.

\section{Material e métodos}

Para alcançar o objetivo proposto foi desenvolvido um estudo do tipo survey descritivo e exploratório, com abordagem quantitativa. A pesquisa foi realizada com uma amostra constituída de auxiliares e técnicos de enfermagem de ambos os sexos, totalizando 50 indivíduos que atuam em área hospitalar, na região do Alto Tietê e que realizam funções relacionadas ao preparo e administração de medicamentos. Como critério de inclusão adotouse as seguintes condições: serem auxiliares e/ou técnicos de enfermagem, atuantes em área hospitalar e terem experiência mínima de seis meses na área hospitalar, especificamente em setores na qual tivessem contato com o processo de administração e preparo de medicações.

A pesquisa teve início após aprovação pelo Comitê de Ética em Pesquisa com Seres Humanos da Universidade de Mogi das Cruzes, no qual obteve parecer favorável para a sua realização sob número 60142 e autorização dos participantes por meio da assinatura do termo de consentimento livre e esclarecido (TCLE).

Para a obtenção dos dados foi aplicado um questionário semiestruturado, elaborado pelos autores do estudo, com base nas literaturas existentes que envolvem e abordam o tema segurança na administração de medicamentos, para atender ao objetivo proposto, sendo aplicado individualmente, mediante explicação prévia do objetivo e metodologia da pesquisa. Esse instrumento foi elaborado com questões relacionadas às dificuldades e formas de melhorias possíveis, que possam ser trabalhadas com a equipe de enfermagem para aumentar e melhorar o entendimento sobre cálculos de medicações. De acordo com o objetivo do estudo e para sistematizar a coleta de dados, o questionário foi dividido em duas partes: caracterização do indivíduo e coleta de dados específicos.

Após a finalização da coleta de dados por meio do instrumento apontado neste estudo, foi realizada uma análise descritiva dos resultados obtidos, no qual as informações relatadas foram representadas por meio de números absolutos e percentuais, sendo demonstradas ainda com o auxílio de tabelas.

Resultados

Entre os 50 profissionais de enfermagem participantes, $62 \%$ eram auxiliares de enfermagem e $38 \%$ técnicos de enfermagem, $80 \%$ eram do sexo feminino e $20 \%$ do sexo masculino, com a idade variando entre 21 e 43 anos, com média de 28,1 anos. A média de tempo de formado foi de 4,9 anos e o tempo de atuação na área da enfermagem variou de 6 meses a 10 anos, com média de 3,4 anos, $50 \%$ dos indivíduos trabalhavam em instituição pública, $40 \%$ em instituição particular e 10\% em instituição filantrópica.

Uma das situações levantadas junto aos participantes foi o conhecimento desses profissionais em relação aos certos que envolvem a prática de aplicação de medicamentos. Percebe-se com os resultados demonstrados na tabela I que a maioria conhece os cinco certos fundamentais da medicação, entretanto nem todos responderam corretamente. A dose certa, que é o enfoque deste estudo devido ao cálculo de medicação, foi o certo mais citado (82\%), o que demonstra, inclusive, a relação do conhecimento dos participantes sobre a importância desse certo, quando relacionado a sua função que é a de garantir a dose adequada para administração ao paciente, sendo citado por $84 \%$ dos indivíduos.

Tabela I - Descrição do conhecimento dos profissionais de enfermagem em relação aos certos da medicação. Mogi das Cruzes/SP, 2013.

\begin{tabular}{lll}
\hline Certos & $\mathrm{n}$ & $\%$ \\
\hline Dose & 41 & 82 \\
Via & 40 & 80 \\
Medicação & 40 & 80 \\
Hora & 37 & 74 \\
Paciente & 37 & 74 \\
\hline
\end{tabular}


Já a dificuldade relacionada ao cálculo de medicação foi relatada por $84 \%$ dos participantes, dentre as citadas, a regra de três, o gotejamento, a transformação de soro e a rediluição de medicamentos foram identificadas como potenciais obstáculos em suas intervenções medicamentosas, diferenciando-se entre si (tabela II).

Tabela II - Descrição das dificuldades relatadas pelos profissionais de enfermagem em relação à realização e o tipo de cálculo de medicação. Mogi das Cruzes/SP,2013.

\begin{tabular}{lllllllllll}
\hline & Nenhuma & $\%$ & Muita & $\%$ & Pouca dificuldade & $\%$ & Não sabe fazer & $\%$ & Em branco & $\%$ \\
\hline Regra de Três & 22 & 44 & 4 & 8 & 14 & 28 & 3 & 6 & 7 & 14 \\
Gotejamento & 10 & 20 & 3 & 6 & 25 & 50 & 3 & 6 & 9 & 18 \\
Transformação de soro & 3 & 6 & 7 & 14 & 8 & 16 & 26 & 52 & 6 & 12 \\
Cálculo de rediluição & 3 & 6 & 18 & 36 & 16 & 32 & 6 & 12 & 7 & 14 \\
\hline
\end{tabular}

Em geral, verificando-se a média dos dados relacionados às respostas sobre as dificuldades em realizar o cálculo de medicação, percebe-se que 19\% dos indivíduos referiram não ter dificuldades, enquanto os demais $81 \%$ apresentaram algum grau de dificuldade na realização dessa atividade, variando de muita dificuldade a pouca dificuldade, não saber fazer a atividade e/ou não ter resposta à situação.

A partir da identificação da opinião dos participantes sobre características que envolvem a prática de cálculo de medicações, esses foram convidados a desenvolverem alguns exercícios (macrogotas, microgotas, $\mathrm{ml} /$ hora, regra de três com Decadron e cálculo de transformação de soro). A tabela III apresenta os acertos e erros referentes a essa atividade proposta.

Tabela III - Descrição dos acertos e erros referentes aos cálculos de medicamentos de acordo com os exercícios propostos. Mogi das Cruzes/ SP, 2013.

\begin{tabular}{lllllllllll}
\hline & Macrogotas & $\%$ & Ml/h & $\%$ & Microgotas & $\%$ & Decadron & $\%$ & $\begin{array}{l}\text { Transformaçã } \\
\text { o de soro }\end{array}$ & $\%$ \\
\hline Correto & 10 & 20 & 6 & 12 & 12 & 24 & 23 & 46 & 3 & 6 \\
Incorreto & 17 & 34 & 11 & 22 & 14 & 28 & 3 & 6 & 6 & 12 \\
Em branco & 23 & 46 & 33 & 66 & 24 & 48 & 24 & 41 & 41 & 82 \\
\hline
\end{tabular}

A média de acertos apresentados na realização dos cálculos foi de $21,6 \%$, já os erros apresentaram média de $20,4 \%$. Além disso, houve um percentual de $58 \%$ dos participantes que deixaram os exercícios em branco, o que pode estar relacionado ao fato de não saberem fazêlos.

A dificuldade para a realização das atividades de cálculos de medicações é multifatorial. Dentre as condições que mais se destacaram e chamaram a atenção está a dificuldade na compreensão da matemática (26\%), associada a cálculos mais complexos como no caso da transformação de soluções (24\%) e falta de conhecimento sobre fórmulas existentes (16\%), além de outros fatores, como dificuldade em entender prescrições médicas, poucas aulas teóricas e falta de cursos gratuitos $(20 \%)$, entretanto houve participantes que relataram não possuir dificuldades (2\%) e alguns não responderam (12\%), conforme apresentado na tabela IV.

Tabela IV - Relato dos principais fatores que dificultam o entendimento a cálculos. Mogi das Cruzes/SP, 2013.

\begin{tabular}{lll}
\hline Opinião & $\mathrm{n}$ & $\%$ \\
\hline Matemática & 16 & 26 \\
Transformação de Soro & 12 & 24 \\
Falta de conhecimento nas fórmulas & 8 & 16 \\
Outros & 10 & 20 \\
Não há dificuldades & 1 & 2 \\
Não responderam & 6 & 12 \\
\hline
\end{tabular}

Uma vez identificadas as condições que desfavorecem a realização do cálculo, a sequência do estudo partiu para levantar junto aos participantes as possibilidades de melhorias na opinião deles. Assim, ao verificar a tabela $V$ são identificadas diversas condições, destacando-se a necessidade de melhorar a clareza referente ao processo de medicação 
(36\%), bem como reforçar os programas de educação continuada (18\%), entre outras possibilidades, juntos aos envolvidos.

Tabela V - Descrição dos fatores que podem possibilitar o melhor entendimento dos cálculos de medicação pelos profissionais de enfermagem. Mogi das Cruzes/SP, 2013.

\begin{tabular}{lll}
\hline Opinião & $\mathrm{n}$ & $\%$ \\
\hline Maior clareza & 18 & 36 \\
Educação continuada & 9 & 18 \\
Maior empenho dos professores & 8 & 16 \\
Maior interesse dos alunos & 6 & 12 \\
Utilizar-se da prática de cálculo manual ao invés de tecnologias & 3 & 6 \\
Não responderam & 6 & 12 \\
\hline
\end{tabular}

Discussão

Entre os cinco certos da administração de medicamentos, o mais relatado entre os participantes foi a dose, portanto, estudos reforçam que os erros envolvidos com doses são mais comuns e apresentam um risco considerável para os pacientes [8].

Observou-se que houve o reconhecimento entre os profissionais de enfermagem sobre a importância do cálculo de medicação em relação à dose, uma vez que sua prática influencia a segurança do paciente e a qualidade do cuidado na prática assistencial. Realizar um cálculo incorreto significa administrar uma dose errada e, consequentemente, traz implicações ao paciente, como a redução do efeito terapêutico, a necessidade de administrar outros medicamentos e, em alguns casos, prolongamento da terapia medicamentosa [10].

A maior parte dos eventos adversos relacionados à administração de medicamentos apresenta a dose medicamentosa como a causa de maior prevalência. Isso demonstra a necessidade de ressaltar a importância dos profissionais em aperfeiçoar seus conhecimentos e habilidades referentes ao cálculo de medicação [10].

Muitas medicações são diluídas e preparadas de maneira inadequada devido à dificuldade dos profissionais em lidar com cálculos matemáticos [11]. Esse fato foi comprovado ao questionar os participantes sobre a existência de dificuldade durante o exercício medicamentoso e como resultado grande parte dos participantes citou possuí-la.

Entre os tipos de cálculos especificados no estudo, a regra de três foi citada como o que apresenta menor dificuldade para realização quando comparada aos demais. A dificuldade no cálculo de gotejamento, assim como o de rediluição e transformação de soro foram relatados como complexos na opinião dos participantes.

Em um estudo realizado com 113 indivíduos, verificou-se que 92\% dos participantes afirmaram pouca ou nenhuma dúvida em relação à regra de três, em contrapartida, $24 \%$ relataram muita dúvida em relação a cálculos com porcentagem [12].

Dentre os fatores que influenciam na realização do cálculo da medicação estão o conhecimento insuficiente quanto à matemática e a falha na educação farmacológica do profissional [13]. Percebe-se, em geral, que a principal barreira que impede a realização de cálculos de medicação efetivamente é a falta de conhecimento de princípios matemáticos básicos que são exigidos durante o cálculo de medicação. Tais deficiências podem levar a dificuldades mesmo com o auxílio de uma calculadora [14].

Aliado à necessidade de conhecer sobre o ponto de vista dos participantes em relação à prática de cálculo de medicações, foi sugerido neste estudo a resolução de alguns exercícios (regra de três, cálculo de infusão e transformação de soluções), com o intuito de correlacionar as respostas obtidas com os resultados relatados na pesquisa.

Ao analisar as respostas, o cálculo de Decadron, que usava a regra de três para a sua resolução, foi o cálculo com mais acertos entre os participantes. Já os exercícios que objetivavam o cálculo de infusão (macrogotas, microgotas e $\mathrm{ml} / \mathrm{h}$ ) e os de transformação de soluções, apresentaram um número elevado de erros, bem como respostas em branco. Demonstrando, assim, os pontos de maior dificuldade entre os profissionais.

O cálculo de porcentagens de medicamentos e as taxas de infusão podem ser conceitualmente mais complicados do que outros cálculos de medicação, e isso explicaria o baixo desempenho nos exercícios [13].

Diante disso, os participantes relataram alguns fatores que comprometem seu entendimento sobre os cálculos. Com isso, a falta de habilidade e conhecimento em matemática associada aos tipos de cálculos conhecidamente mais complexos, como no caso 
da transformação de soluções e de infusão, a falta de conhecimento em fórmulas, entre outras condições podem ser considerados como contribuintes para falhas e limitações.

A dificuldade evidenciada em matemática na área da enfermagem tem forte relação com a multiplicação de frações, seguido por interpretação de informações, proporções, posição de valor, frações e porcentagens [14]. Aliado a isso, uma situação que pode corroborar essa condição pode estar atrelada ao aumento no uso de tecnologias, como, por exemplo, bombas de infusão com contadores de taxa de gotejamento que podem contribuir na perda de habilidades dos profissionais [13].

Dentre as sugestões relacionadas a melhorias no processo de entendimento dos profissionais em relação aos cálculos, na opinião dos participantes, percebeu-se a necessidade de buscar maior clareza, bem como o reforço em educação continuada, corroborando o entendimento e capacitando os envolvidos no processo da terapia medicamentosa.

É necessário que as instituições busquem identificar as habilidades matemáticas e o nível de conhecimento sobre farmacologia de seus profissionais, a fim de elaborar estratégias para manter a equipe de enfermagem atualizada, por meio de programas de capacitação [15]. A educação continuada, neste contexto, possibilita o aprimoramento do conhecimento técnicocientífico e prático, melhora a comunicação entre os profissionais envolvidos e corrobora na redução e prevenção dos erros no processo de medicação. Esse conhecimento garante uma maior qualidade da assistência, a confiança da equipe e uma prática humanizada e fundamentada cientificamente [2].

Contudo, a adoção de práticas profissionais baseadas em protocolos e evidências clínicas, assim como a abertura para se aprender a partir de falhas, ajuda a tornar a assistência hospitalar mais segura para o paciente [17]. Dessa forma, reconhecer a causa raiz do erro é necessário para se identificar onde e como intervir [18].

Deve ser destacada a importância na formação acadêmica dos profissionais de enfermagem em relação aos cálculos de medicação, utilizando-se, ainda, da educação continuada por meio de cursos de atualização, palestras e capacitações dos indivíduos que estejam envolvidos com o preparo e administração de medicamentos, pois, além de diminuir os erros de dosagem garante-se a segurança da assistência ao paciente.

Conclusão

O processo de medicação é uma das funções de maior responsabilidade da equipe de enfermagem, e erros durante esse processo podem ocasionar danos graves ao paciente, além de consequências sérias ao profissional e à instituição de saúde. Assim, este estudo permitiu identificar as dificuldades encontradas pelos profissionais em realizar cálculos de medicações e os pontos a serem melhorados para que os erros sejam minimizados ou prevenidos.

Diante dos resultados alcançados, o fator que mais dificulta a realização dos cálculos é a falta de conhecimento dos participantes em relação à matemática, bem como sobre a compreensão do que se necessita. Dessa maneira, o cálculo incorreto da dosagem a ser administrada interfere no processo dos cinco certos que auxiliam uma assistência sem erros de medicações. Assim, ressalta-se que a falta de conhecimento em matemática gera desconhecimento nas fórmulas, impedindo o cálculo da dosagem correta dos fármacos, e o processo de medicação se torna ineficaz.

Percebe-se que a limitação encontrada na realização desta pesquisa direciona-se a falta de estudos voltados a essa temática, pois muito se aborda sobre a segurança do processo de medicação, mas dificilmente são realizados estudos que abordam a dificuldade da equipe de enfermagem como um todo em relação ao processo de cálculo de medicações, que também faz parte dessa atividade.

Diante disso, conclui-se que existe a necessidade de reforçar os programas de educação continuada no sentido de favorecer uma maior segurança e assertividade nesse processo na assistência e enfermagem. Espera-se que a realização deste estudo venha a contribuir para que profissionais e instituições direcionem atenção para a minimização das condições de risco que possam estar associadas a esse processo.

Referências

1. Aizenstein ML. Fundamentos para o uso racional de medicamentos. $3^{\underline{a}}$ ed. São Paulo: Artes Médicas; 2016. 
2. Nascimento MA, Freitas K, Oliveira CGS. Erros na administração de medicamentos na prática assistencial da equipe de enfermagem: uma revisão sistemática. Cadernos de Graduação Ciências Biológicas e Saúde 2016;3(3):241-56.

3. Ferreira MM, Alves FS, Jacobina FB. O profissional de enfermagem e a administração segura de medicamentos. Revista Enfermagem Contemporânea 2014;3(1):61-9. https://doi.org/10.17267/2317-3378rec.v3i1.208.

4. Cassiani SHB, Oliveira RC. Administração de medicamentos, erros de medicação e segurança do paciente. Revista Mineira de Enfermagem 2011;15(2):163-4.

5. NCCMERP. National Coordinating Council for Medication Error Reporting and Prevention. About medication errors. [Internet] 2016 [citado 2017 Jan 18]. Disponível em: http://www.nccmerp.org/about-medication-errors.

6. BAHIA. Parecer Coren - BA N033/2014. Conselho Regional de Enfermagem de Bahia [Internet] 2014 [citado 2018 Jan 31]. Disponível em: http://ba.corens.portalcofen.gov.br/parecer-coren-ba-n\%E2\%81\%B00332014_15634.html

7. São Paulo. Uso seguro de medicamentos: guia para preparo, administração e monitoramento. Conselho Regional de Enfermagem de São Paulo [Internet] 2017 [citado 2018 Jan 16]. Disponível em: http://portal.coren-sp.gov.br/sites/default/files/usoseguro-medicamentos.pdf.

8. Silva JSD, Almeida PHRF, Perini E, Pádua CAM, Rosa MB, Lemos GS. Erros de prescrição e administração envolvendo um medicamento potencialmente perigoso. Rev de Enfermagem 2017;11(10):3707-17.

9. Potter P, Perry AG. Fundamentals of Nursing. 9 ${ }^{\mathrm{a}}$ ed. St Louis: Elsevier; 2017.

10. Sailabala M. Awareness of medication error, medication management and prevention among staff nurses in IMS \&Sum Hospital, Odisha. Nitte University Journal of Health Science 2016;6(4):18-22.

11. Galiza DDF, Moura OF, Barros VL. Preparo e administração de medicamentos: erros cometidos pela equipe de enfermagem. Rev Bras Farm Hosp Serv Saúde São Paulo 2014;5(2):45-50.

12. Pereira CMB, Pereira OB, Carboni RM. Conduta do futuro enfermeiro mediante o erro de medicação. Rev Enferm UFPE on line 2010;4(1):118-23.

13. Ofosu $R$, Jarrett $P$. Reducing nurse medicine administration erros. Nursing Times 2015;111(20):12-15.

14. Wright K. Barriers to accurate drug calculations. Nursing Standard 2006;20(28):41-5. https://doi.org/10.7748/ns2006.03.20.28.41.c4099

15. Fleming S, Brady AM, Malone AM. An evaluation of the drug calculation skills of registered nurses. Nurse Education in Practice 2014;14(1):55-61.

16. Duarte LMCPS, Medeiros WR, Martins QCS. Administração segura de medicamentos: a educação permanente como estratégia de promoção [TCC]. Natal: Universidade do Rio Grande do Norte; 2015.

17. Vilela RPB, Jericó MC. Erro de medicação: gestão do indicador para uma prática mais segura. Rev Enferm UFPE 2016;10(1):119-27. 